\title{
Is Receptor-Interacting Protein Kinase 3 a Viable Therapeutic Target for Mycobacterium tuberculosis Infection?
}

\author{
Michael D. Stutz ${ }^{1,2}$, Samar Ojaimi ${ }^{1,2}$, Gregor Ebert ${ }^{1,2}$ and Marc Pellegrini ${ }^{1,2 *}$ \\ 1 Infection and Immunity Division, The Walter and Eliza Hall Institute of Medical Research, Melbourne, VIC, Australia, \\ ${ }^{2}$ Department of Medical Biology, The University of Melbourne, Melbourne, VIC, Australia
}

OPEN ACCESS

Edited by:

Olivier Neyrolles,

Centre national de la

recherche scientifique

(CNRS), France

Reviewed by:

Christophe J. Queval,

Francis Crick Institute,

United Kingdom

Selvakumar Subbian,

Public Health Research

Institute (PHRI),

United States

*Correspondence:

Marc Pellegrin

pellegrini@wehi.edu.au

Specialty section:

This article was submitted to Molecular Innate Immunity,

a section of the journal

Frontiers in Immunology

Received: 01 February 2018

Accepted: 11 May 2018

Published: 28 May 2018

Citation:

Stutz MD, Ojaimi S, Ebert G and Pellegrini M (2018) Is

Receptor-Interacting Protein

Kinase 3 a Viable Therapeutic

Target for Mycobacterium tuberculosis Infection?

Front. Immunol. 9:1178.

doi: 10.3389/fimmu.2018.01178
The dwindling list of antimicrobial agents exhibiting broad efficacy against clinical strains of Mycobacterium tuberculosis (Mtb) has forced the medical community to redefine current approaches to the treatment of tuberculosis (TB). Host receptor-interacting protein kinase 3 (RIPK3) has been flagged recently as a potential target, given that it is believed to regulate necroptosis-independent signaling pathways, which have been implicated in exacerbating several inflammatory conditions and which reportedly play a role in the necrosis of Mtb-infected macrophages. To examine the therapeutic potential of inhibiting RIPK3, we infected RIPK3-deficient mice with aerosolized Mtb. We found that the loss of RIPK3 did not alter overall disease outcomes, with deficient animals harboring similar bacterial numbers in the lungs and spleens compared to their wild-type counterparts. Mtb-infected macrophages were not rescued from dying by Ripk3 deletion, nor did this affect production of the pro-inflammatory cytokine IL-1 $\beta$, both in vitro and in vivo. Infiltration of immune cells into the lungs, as well as the activation of adaptive immunity, similarly was not overtly affected by the loss of RIPK3 signaling. Collectively, our data argue against a role of RIPK3 in mediating pathological inflammation or macrophage necrosis during Mtb disease pathogenesis and thus suggest that this host protein is unlikely to be an attractive therapeutic target for TB.

Keywords: receptor-interacting protein kinase 3, tuberculosis, macrophage, necrosis, host-directed therapy, inflammation

\section{INTRODUCTION}

The alarmingly rapid emergence of highly drug-resistant strains of Mycobacterium tuberculosis $(\mathrm{Mtb})$ has given traction to therapeutic approaches that target host cellular processes rather than the pathogen itself. Investigations of these so-called host-directed therapies have largely focused on modulating the function of the macrophage to Mtb infection, given that this innate immune player is a principal host cell involved in the immunological response to tuberculosis (TB).

The receptor-interacting protein kinase (RIPK) family comprises seven members whose defining feature is a homologous serine/threonine kinase domain, but which differ widely in terms of functional domains (1). As their name implies, the RIPK proteins play fundamental roles in the response to intracellular and extracellular stimuli and have emerged as critical regulators of inflammatory and cell death signaling pathways, leading to intense interest in pharmacologically targeting 
these proteins (1-3). Although the functions of RIPK4-7 are poorly understood, RIPK2 is known to be required for signaling following detection of bacterial peptidoglycan derivatives by the NOD2 cytosolic receptor (3). Studies of RIPK1 have elucidated its pivotal role in dictating the outcome of death receptor signaling, in terms of whether a cell triggers pro-inflammatory gene expression or engages the apoptotic cell death pathway. However, RIPK1 also interacts with RIPK3, the best characterized role of which is the phosphorylation of mixed lineage kinase domainlike (MLKL) - a dedicated pseudokinase that is essential for the execution of a regulated necrotic form of cell death, termed necroptosis $(4,5)$.

We recently demonstrated that Mtb-infected human and mouse macrophages do not undergo necroptosis and that this form of cell death does not contribute to Mtb disease pathogenesis in vivo (6). However, phenotypic differences between Ripk $3^{-/-}$ and $M l k l^{-/-}$mice (both of which are unable to engage necroptosis) in numerous disease models have made it clear that RIPK3 participates in additional pathways beyond inducing necroptosis (7). Several reports have now described MLKL-independent roles of RIPK3 in pro-inflammatory signaling in macrophages and dendritic cells, including NLRP3 inflammasome activation and IL-1 $\beta$ maturation (8-13), and more recently, the production of type I IFN $(14,15)$. Such pathways reportedly contribute to protection against certain infections such as influenza A virus, with $R i p k 3^{-/-}$ mice experiencing a heightened susceptibility to infection due to an inability of macrophages to produce type I IFN in the lungs $(10,14)$. The cell death-independent functions of RIPK3 have also been implicated in the pathogenesis of several diseases. For example, Ripk3 deletion was found to attenuate disease in mouse models of kidney ischemia-reperfusion injury, myocardial infarction, and systemic inflammation (7). RIPK3-dependent inflammasome activation and IL- $1 \beta$ secretion by macrophages were also shown to be responsible for retinal detachment-induced photoreceptor death (16). Type I IFNs are generally associated with TB disease progression (17-19). Although IL-1 $\beta$ is essential for the control of Mtb $(20,21)$, and the NLRP3 inflammasome appears to be dispensable for its production (22), individuals carrying a polymorphism in the $I L 1 B$ promoter region that increases IL-1 $\beta$ expression are more susceptible to severe active TB disease with extrapulmonary lesions (23). Intriguingly, two groups recently reported that RIPK3 promotes necrosis of Mtb-infected macrophages in vitro by stimulating the production of mitochondrial reactive oxygen species (ROS), via a pathway requiring the anti-apoptotic protein BCL-XL $(24,25)$. These experiments, which largely used RNAi and human and immortalized murine macrophages, were complemented with in vivo studies, in which Ripk3-deficient mice were shown to harbor fewer bacteria in the lungs following high-dose intravenous Mtb infection, although this was substantially less striking when mice were infected by low-dose aerosol (25). The authors concluded that RIPK3 signaling is thus pathogenic during $\mathrm{Mtb}$ infection by promoting the escape of mycobacteria from necrotic macrophages and facilitating dissemination, leading to worse disease outcomes.

Reports that RIPK3-dependent inflammatory signaling by macrophages is pathological in many infectious and non-infections disease models, and recent work concluding that RIPK3 mediates a necrotic macrophage death modality, have emphasized the potential therapeutic benefit of targeting this host protein for the treatment of TB. The development of small molecule inhibitors of RIPK3 is being aggressively pursued $(26,27)$ with several such compounds undergoing testing in preclinical models of disease. It is therefore of utmost importance to clearly define the involvement of RIPK3 in the pathogenesis of Mtb infection. Our previous work demonstrating that the MLKL-dependent necroptosis does not contribute to Mtb disease has enabled us to specifically examine the non-necroptotic functions of RIPK3 by using mice genetically targeted for this gene.

\section{MATERIALS AND METHODS}

\section{Mice}

The Walter and Eliza Hall Institute of Medical Research Animal Ethics Committee reviewed and approved all animal experiments. Six- to 10-week old male and female C57BL/6, Ripk3 ${ }^{-/-}$(28)

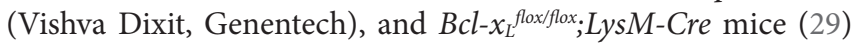
(James Vince, WEHI) were used and were age- and sex-matched in all experiments. Mice infected with Mtb were housed in individually ventilated microisolator cages.

\section{Bacteria}

Mycobacterium tuberculosis strain $\mathrm{H} 37 \mathrm{Rv}$ and a strain of $\mathrm{H} 37 \mathrm{Rv}$ episomally expressing mCherry were both sourced from Nicholas P West (University of Queensland). Mycobacteria were cultured in albumin-dextrose-catalase supplemented 7H9 medium (BD Biosciences, San Jose, CA, USA) and prepared as a single-cell suspension as described previously (6). Briefly, bacteria were pelleted and washed with PBS $+0.05 \%$ Tween-80 (Sigma-Aldrich), before low-speed centrifugation at $130 \times g$ for $8 \mathrm{~min}$ to pellet aggregated bacteria. Single-cell bacteria in the supernatant were quantitated by measuring optical density (OD; $590 \mathrm{~nm}$ ), with an $\mathrm{OD}_{590} 0.1$ estimated to be $5 \times 10^{7} \mathrm{CFU} / \mathrm{ml}$. The mCherry-expressing strain was used in some experiments as indicated and was cultured in the presence of $25 \mu \mathrm{g} / \mathrm{ml}$ kanamycin (Sigma-Aldrich).

\section{BCL-XL Inhibitor}

In some experiments, mice were administered $25 \mathrm{mg} / \mathrm{kg}$ of the BCL-XL-selective inhibitor A-1331852 (30) (AbbVie Inc., North Chicago, IL, USA) by daily oral gavage. Control mice received an equivalent volume of vehicle, which consisted of $60 \%$ Phosal 50 PG (Lipoid), 27.5\% PEG400 (Affymetrix), 10\% ethanol, and 2.5\% DMSO (Ajax Finechem). Platelet counts in the blood of treated mice were determined using an ADVIA 2120 hematology analyzer (Siemens Australia New Zealand, VIC, Australia).

\section{Macrophage Isolation and Infection}

Bone marrow-derived macrophages (BMDMs) were prepared from WT and $R i p k 3^{-/-}$mice as described previously (6). Differentiated BMDMs were plated at a density of $4 \times 10^{5}$ cells per well (12-well plates) in antibiotic-free DMEM supplemented with 10\% fetal bovine serum (Sigma-Aldrich) and 15\% L929conditioned medium, which was maintained for the entire duration of the experiment. Cells were treated/infected at various 
multiplicities of infection (MOIs) as indicated $24 \mathrm{~h}$ after plating by adding single-cell Mtb suspensions into the culture.

\section{Cell Death Analysis}

Adherent and non-adherent BMDMs were collected 48 h postinfection, stained with $25 \mu \mathrm{g} / \mathrm{ml}$ propidium iodide (SigmaAldrich) and analyzed using an LSRFortessa X-20 flow cytometer (BD Biosciences).

\section{Western Blots}

Bone marrow-derived macrophages were harvested $24 \mathrm{~h}$ after infection with Mtb and/or treatment with $50 \mathrm{ng} / \mathrm{ml} \mathrm{recombinant}$ mouse IFN $\gamma$ (Biolegend). Proteins were isolated using cell lysis buffer containing 1\% Triton X-100 (Sigma-Aldrich), $20 \mathrm{mM}$ Tris- $\mathrm{HCl}, \mathrm{pH}$ 7.5, $135 \mathrm{mM} \mathrm{NaCl}, 1.5 \mathrm{mM} \mathrm{MgCl}, 1 \mathrm{mM}$ EGTA, $10 \%$ glycerol, EDTA-free protease inhibitor tablets (Roche), and phosphatase inhibitor tablets (Roche). Equal quantities (30 $\mu \mathrm{g}$ ) of total protein were separated under denaturing and reducing conditions using 4-12\% SDS-PAGE gels (Life Technologies), transferred onto nitrocellulose membranes, blocked with 5\% skim milk for $1 \mathrm{~h}$, and detected using the following primary antibodies: rabbit anti-iNOS (cat. \#ab178945; Abcam), rabbit anti-RIP3 (cat. \#2283; ProSci; RRID: AB_203256), and rabbit anti- $\beta$-actin-HRP (cat. \#5125; Cell Signaling Technology; RRID: AB_1903890). HRP-conjugated goat secondary antibodies (Southern Biotech) were applied to membranes, which were then incubated with Luminata Forte Western HRP substrate (Merck) and imaged using a ChemiDoc Touch Imaging System (Bio-Rad). Densitometry was performed using Image Lab v.5.2.1 software (Bio-Rad; RRID:SCR_014210).

\section{Cytokine Analysis}

Bone marrow-derived macrophages were infected with Mtb in the presence of GolgiStop and GolgiPlug (BD Biosciences) for $4 \mathrm{~h}$. Cells were then harvested, fixed with $4 \%$ paraformaldehyde (PFA) for $30 \mathrm{~min}$, permeabilized (Perm/Wash Buffer, BD Biosciences), and stained for intracellular TNF by incubating cells with rat anti-TNF PE (clone: MP6-XT22; Biolegend; RRID: AB_315427) for $45 \mathrm{~min}$ at $4^{\circ} \mathrm{C}$. Stained cells were analyzed by flow cytometry.

Bone marrow-derived macrophage culture supernatants were centrifuged at $500 \times g$ for $5 \mathrm{~min}$, filter-sterilized $(0.22 \mu \mathrm{m})$, and analyzed for TNF and IL-1 $\beta$ using DuoSet ELISA kits (R\&D Systems), according to the manufacturer's instructions. Lung tissue homogenates (described below) were diluted 1:1 in cell lysis buffer containing protease inhibitors, centrifuged at $9,000 \times g$ for $10 \mathrm{~min}$ to pellet debris, and analyzed for TNF, IL-1 $\beta$, and IFN $\gamma$.

\section{Aerosol Infection and Quantitation of Mtb}

Mice were infected with Mtb by low-dose aerosol ( 100-200 CFU), using a whole-body Inhalation Exposure System (Glas-Col), as described previously (6). Three mice were sacrificed $24 \mathrm{~h}$ after aerosol exposure to confirm the pulmonary infection dose.

Mice were euthanized at various times post-infection by $\mathrm{CO}_{2}$ asphyxiation, and organs were collected and homogenized with steel beads in PBS $+0.05 \%$ Tween- 80 using a Bullet Blender
(Next Advance, Inc.). Homogenates were serially diluted and plated onto Middlebook 7H11 agar (BD Biosciences) supplemented with $0.5 \%(\mathrm{v} / \mathrm{v})$ glycerol and $10 \%(\mathrm{v} / \mathrm{v})$ OADC supplements. Colonies were counted after 21 days incubation at $37^{\circ} \mathrm{C}$ and were expressed as CFU/organ.

\section{Histology}

Lungs were inflated by intratracheal perfusion with $4 \%$ PFA and fixed overnight at $4^{\circ} \mathrm{C}$. Left lobes were embedded in paraffin, sectioned, and stained with hematoxylin and eosin. Slides were scanned with an Aperio ScanScope AT slide scanner (Leica Microsystems) and images analyzed using FIJI software with custom-written macros.

\section{Bronchoalveolar Lavage (BAL)}

Mice were euthanized, and the trachea cannulated with an 18-gauge needle. BAL was performed with eight $1 \mathrm{ml}$ aliquots of ice-cold Hank's Balanced Salt Solution (Ca-/Mg-free) containing $0.5 \mathrm{mM}$ EDTA. Retrieved cells were cultured in tissue culture-treated plates for $45 \mathrm{~min}$ to allow alveolar macrophages to adhere. Unattached cells were removed, and plates washed with PBS. Macrophages were detached with trypsin-EDTA (Sigma-Aldrich), washed with PBS, and counted using a Neubauer hemocytometer. Cells were then lysed with $0.5 \%$ Triton X-100 in PBS, serially diluted, and plated as described above to quantitate intracellular CFU.

\section{Immune Cell Profiling and Peptide Restimulation}

Immune cells were isolated from the lungs and spleens of infected mice as described previously (6). A portion of isolated lung cells and splenocytes were stained with the following antibodies (all from $\mathrm{BD}$ Biosciences) for $45 \mathrm{~min}$ at $4^{\circ} \mathrm{C}$ : rat anti-CD16/ CD32 (RRID: AB_394657), rat anti-CD4 BV421 (clone: GK1.5), rat anti-CD8 PE-Cy7 (clone: 53-6.7; RRID: AB_394506), rat anti-CD19 PerCP-Cy5.5 (clone: 1D3; RRID: AB_394004), rat anti-CD11b BV510 (clone: M1/70), hamster anti-CD11c APC (clone: HL3; RRID: AB_398460), rat anti-I-A/I-E (clone: 2G9; RRID: AB_394958) FITC, rat anti-Ly-6G/Ly-6C PE (clone: RB6-8C5; RRID: AB_394644), rat anti-PD1 PE (clone: J43; RRID: AB_394284), and hamster anti-CD69 BV421 (clone: H1.2F3; RRID: AB_2687478). Cells were washed and fixed with 4\% PFA for $30 \mathrm{~min}$.

Approximately $2 \times 10^{6}$ splenocytes and $3 \times 10^{6}$ lung cells were cultured in Iscove's modified Dulbecco's medium containing $10 \%$ FCS, $\beta$-mercaptoethanol, penicillin/streptomycin, GolgiStop, and GolgiPlug. Cells were stimulated with $5 \mu \mathrm{g} / \mathrm{ml} \mathrm{Mtb}$ early secreted antigenic target 6 (ESAT-6; MTEQQWNFAGIEAAA) peptide (GenScript), and incubated for $5 \mathrm{~h}$. Cells were then harvested, surface stained with rat anti-CD4 BV421 and rat antiCD8 PE-Cy7, fixed with 4\% PFA for $30 \mathrm{~min}$, and permeabilized. Intracellular cytokine staining was performed by incubating cells with rat anti-TNF PE (clone: MP6-XT22; Biolegend) and rat anti-IFN $\gamma$ FITC (clone: XMG1.2; BD Biosciences; RRID: AB_395375) for $45 \mathrm{~min}$ at $4^{\circ} \mathrm{C}$. Stained cells were analyzed by flow cytometry. 


\section{Statistics}

Prism 6.0h (GraphPad Software) was used to perform statistical tests. Groups were compared by unpaired two-tailed $t$ tests for parametric data. Non-parametric data were log-transformed for statistical analysis, or when this failed to normalize the data, Mann-Whitney tests were used. Holm-Sidak correction was applied for multiple $t$ tests.

\section{RESULTS}

\section{Macrophage Responses to Mtb Infection Are Unaltered by the Loss of RIPK3}

Macrophages are the principal host cells involved in the immune response to Mtb infection. We therefore addressed whether the loss of the RIPK3 signaling axis altered any aspects of the antimycobacterial response of murine macrophages.

First, we considered the possibility that the loss of RIPK3 signaling may affect the ability of macrophages to internalize Mtb. Differences in the initial bacterial burden would confound interpretation of our subsequent experiments. To address this, we infected BMDMs derived from wild-type (WT) and Ripk3 $3^{-/}$ mice with an mCherry-expressing Mtb strain and analyzed mCherry fluorescence in BMDMs by flow cytometry $4 \mathrm{~h}$ after infection. The proportion of cells harboring intracellular mycobacteria (mCherry fluorescence) was similar between WT and Ripk $3^{-/-}$BMDMs at all MOIs (Figure 1A). The median fluorescence intensity of the mCherry signal-a marker of intracellular bacterial numbers-was comparable between genotypes, albeit marginally lower in Ripk $3^{-/-}$cells infected at higher MOI (Figure 1B).

We next examined the macrophage response to intracellular Mtb, which involves the secretion of a number of cytokines including TNF and IL-1 $\beta$, as well as the activation of microbial killing mechanisms such as inducible nitric oxide synthase (iNOS). These components of the immune response are indispensable during infection in vivo $(20,31,32)$. We found that the loss of RIPK3 did not alter the proportion of BMDMs producing TNF following Mtb infection, as determined by intracellular cytokine staining (Figure 1C). Additionally, the quantities of TNF and
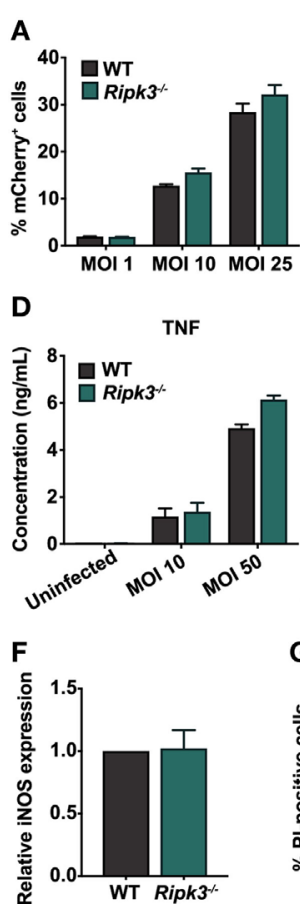
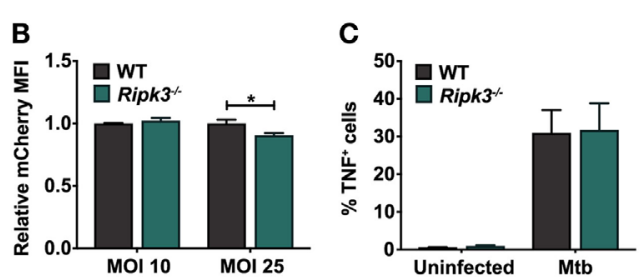

E

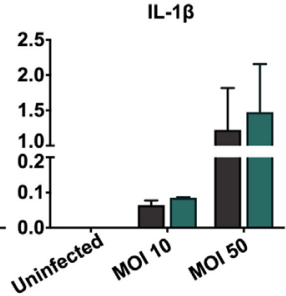

G

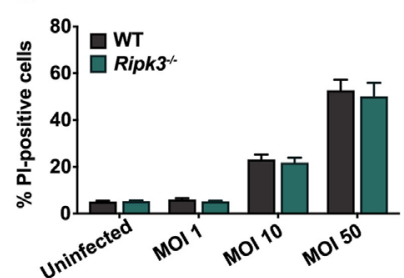

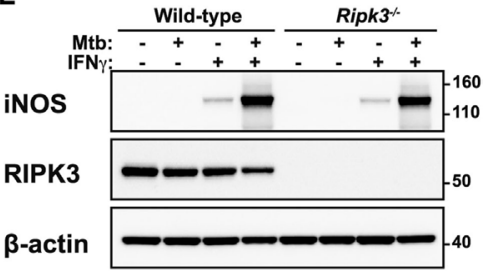

H

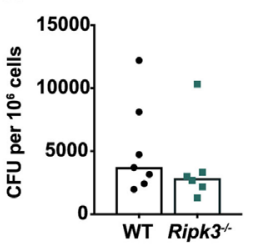

FIGURE 1 | Responses of murine macrophages to Mycobacterium tuberculosis (Mtb) infection are unaltered by the loss of receptor-interacting protein kinase 3 (RIPK3). (A) Percentage of total bone marrow-derived macrophages (BMDMs) positive for mCherry fluorescence. Cells were infected with mCherry-expressing Mtb H37Rv for $4 \mathrm{~h}$ at the indicated multiplicities of infection (MOls). (B) Relative median fluorescence intensity (MFl) of mCherry in mCherry-positive cells in (A). (C) Percentage of total BMDMs producing TNF in response to Mtb infection. Cells were infected with Mtb for $4 \mathrm{~h}$ at an MOI of 25 and analyzed by intracellular cytokine staining and flow cytometry. (D) Concentrations of TNF and IL-1 $\beta$ in culture supernatants of WT and Ripk3 ${ }^{-/-}$BMDMs $24 \mathrm{~h}$ post-infection with various MOls of Mtb, determined by ELISA. (E) Western blot of inducible nitric oxide synthase (iNOS) expression in wild-type (WT) and Ripk3-/- BMDMs infected with Mtb (MOI 10) and/or treated with IFN $\gamma(50 \mathrm{ng} / \mathrm{ml})$. Numbers to the right of the panels represent the positions of protein size markers (in $\mathrm{kDa}$ ). (F) Densitometric quantitation of relative iNOS protein expression in BMDMs infected with Mtb and primed with IFN $\gamma$ (adjusted for actin loading). (G) Death of WT and Ripk3 ${ }^{-/-}$BMDMs $48 \mathrm{~h}$ after infection with various MOls of Mtb. The amount of cell death was determined by propidium iodide staining and flow cytometry. (H) Intracellular bacterial numbers in alveolar macrophages isolated from the bronchoalveolar lavage (BAL) of WT and Ripk3 ${ }^{-/-}$mice 4 weeks post-infection. Each point represents one mouse $(n=6-7)$.

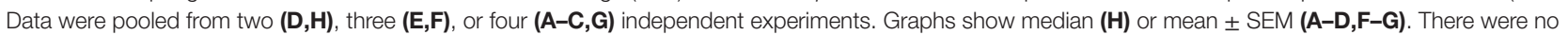
statistically significant differences between genotypes $(p>0.05)$ unless indicated. ${ }^{*} p<0.05$. 
IL-1 $\beta$ secreted into the tissue culture supernatant over a $24 \mathrm{~h}$ period following infection were comparable between genotypes (Figure 1D). We also did not observe any difference in the ability of Ripk3 $3^{-/-}$BMDMs to express iNOS upon infection (Figures 1E,F). Despite activation of antimicrobial responses following exposure to Mtb, most infected macrophages ultimately die, and RIPK3 has been implicated as a mediator of necrotic macrophage death $(24,25)$. However, we found no differences between WT and Ripk $3^{-/-}$BMDMs in terms of the proportion of cells dying in response to Mtb infection at various MOIs (Figure 1G).

Receptor-interacting protein kinase 3 deficiency did not result in any defects in terms of Mtb internalization or activation of antimicrobial defenses, suggesting that during infection in vitro, RIPK3 does not impact on the capacity of macrophages to respond to intracellular Mtb. However, a multitude of factors contribute to the macrophage response in vivo, which cannot be modeled in vitro. Thus, to determine whether RIPK3 deficiency impacted on intracellular Mtb burdens during physiological infection in vivo, we isolated alveolar macrophages from the lungs of Mtb-infected mice by BAL and assessed intracellular CFU by plating cell lysates. However, there was no significant difference in intracellular bacterial numbers between genotypes (Figure 1H).

\section{The Loss of RIPK3 Signaling Ultimately Does Not Alter Disease Outcomes In Vivo}

Abrogating RIPK3 signaling in macrophages by deleting Ripk3 did not promote anti-mycobacterial activity or prevent macrophage death in vitro. However, we considered the possibility that macrophage responses in the context of Ripk 3 deletion may differ in the presence of other immune cells and cytokines that are involved in infection in vivo. Thus, we examined whether targeting RIPK3 signaling in vivo promoted the clearance of Mtb by using Ripk $3^{-/-}$mice and a physiologically relevant aerosol model of infection. We found that the bacterial burdens in the lungs of Ripk $3^{-/-}$mice were indistinguishable from their WT counterparts 10 days post-infection (Figure 2A). Surprisingly, we noted a small increase in bacterial number 4 weeks post-infection, which correlated with a very mild increase in the size of inflammatory lesions in the lung (Figures 2A,C,D). However, this slight worsening of disease in Ripk $3^{-/}$mice at 4 weeks post-infection was not observed at a later time point (Figure 2A), with all mice surviving the entire duration of the study. Bacterial burdens in the spleen at all time points were indistinguishable between genotypes (Figure 2B).

Receptor-interacting protein kinase 3 was reported to promote macrophage necrosis and therefore disease progression in a process dependent on BCL-XL (25). This would imply that deletion or inhibition of BCL-XL would prevent necrosis and reduce disease severity. However, we found that bacterial burdens in the lungs and spleens of mice deficient for BCL-XL specifically in myeloid cells were indistinguishable from WT littermate controls (Figure 3A). To examine the therapeutic potential of targeting BCL-XL, which may be masked when animals are deficient in this protein from ontogeny, we used the BCL-XL-selective inhibitor A-1331852 to treat Mtb-infected mice (30). We confirmed in vivo pharmacologic activity of A-1331852 by demonstrating a significant reduction in platelet numbers in a cohort of naïve mice treated with the inhibitor (Figure S1 in Supplementary Material). Platelet survival depends on BCL-XL (33), and its inhibition thus results in thrombocytopenia (30), which confirms on-target BCL-XL inhibition. However, 4-week-infected mice treated with 10 doses of A-1331852 over a 2-week period again did not differ from vehicle-treated mice in terms of pulmonary and splenic Mtb bacterial numbers (Figure 3B).

\section{Immunological Responses to Mtb Are Unaffected by the Loss of RIPK3}

Although we did not observe any improvement in Mtb disease outcomes by deleting Ripk3, it remained plausible that any benefit conferred to macrophages by the loss of RIPK3, as the literature would suggest, was nullified by detrimental changes in the function of other immune cells. We thus examined whether there were any differences in immunological responses to $\mathrm{Mtb}$ infection in $R i p k 3^{-/-}$versus WT mice at 4 weeks post-infection. First, the number of macrophages in the lungs and spleens was similar between genotypes (Figures 4A,E). Overall, there were also no statistically significant differences in the number of other innate or adaptive immune cells including granulocytes, dendritic cells, T cells, and B cells (Figures 4A,B), or in the number or percentage of Mtb ESAT-6-specific (IFN $\gamma / \mathrm{TNF}$-producing) $\mathrm{CD}^{+} \mathrm{T}$ cells in the lungs of infected mice (Figure 4C). Consistent with these results, the concentrations of TNF, IL-1 $\beta$, and IFN $\gamma$ in lung homogenates were comparable between genotypes (Figure 4D).

However, although there were no statistically significant differences in immune/inflammatory markers, we did observe slight trends toward increased number of granulocytes, CD4 ${ }^{+} \mathrm{T}$ cells, and acutely activated $\left(\mathrm{CD} 9^{+}\right) \mathrm{CD}^{+}$and $\mathrm{CD}^{+} \mathrm{T}$ cells in the lungs of Ripk $^{-/-}$mice (Figures 4A,B,F,G).

\section{DISCUSSION}

The identification of necroptosis-independent roles of RIPK3 in driving counterproductive inflammatory responses, as well as very recent reports of a role for this protein in the necrosis of Mtb-infected macrophages, has led to the suggestion that RIPK3 may represent a therapeutic target for TB. However, our data challenge this notion and show convincingly that targeting host RIPK3 does not represent a viable therapeutic option for Mtb infection.

Using a low-dose aerosol model of Mtb infection of mice, which mimics the natural acquisition of this pathogen by humans, we showed that the loss of Ripk3 did not fundamentally alter disease outcomes in Mtb-infected mice. Our results were somewhat surprising in light of recent reports suggesting that RIPK3 signaling is pathogenic during Mtb infection due to its induction of a necrotic form of macrophage death, which promotes bacterial dissemination $(24,25)$. The authors described a $0.5 \log$ decrease in bacterial numbers in the lungs of $\mathrm{Ripk}^{-/-}$mice 4 weeks postinfection, in a high-dose intravenous challenge model (25). It is unclear, however, whether this phenotype persisted at later time 

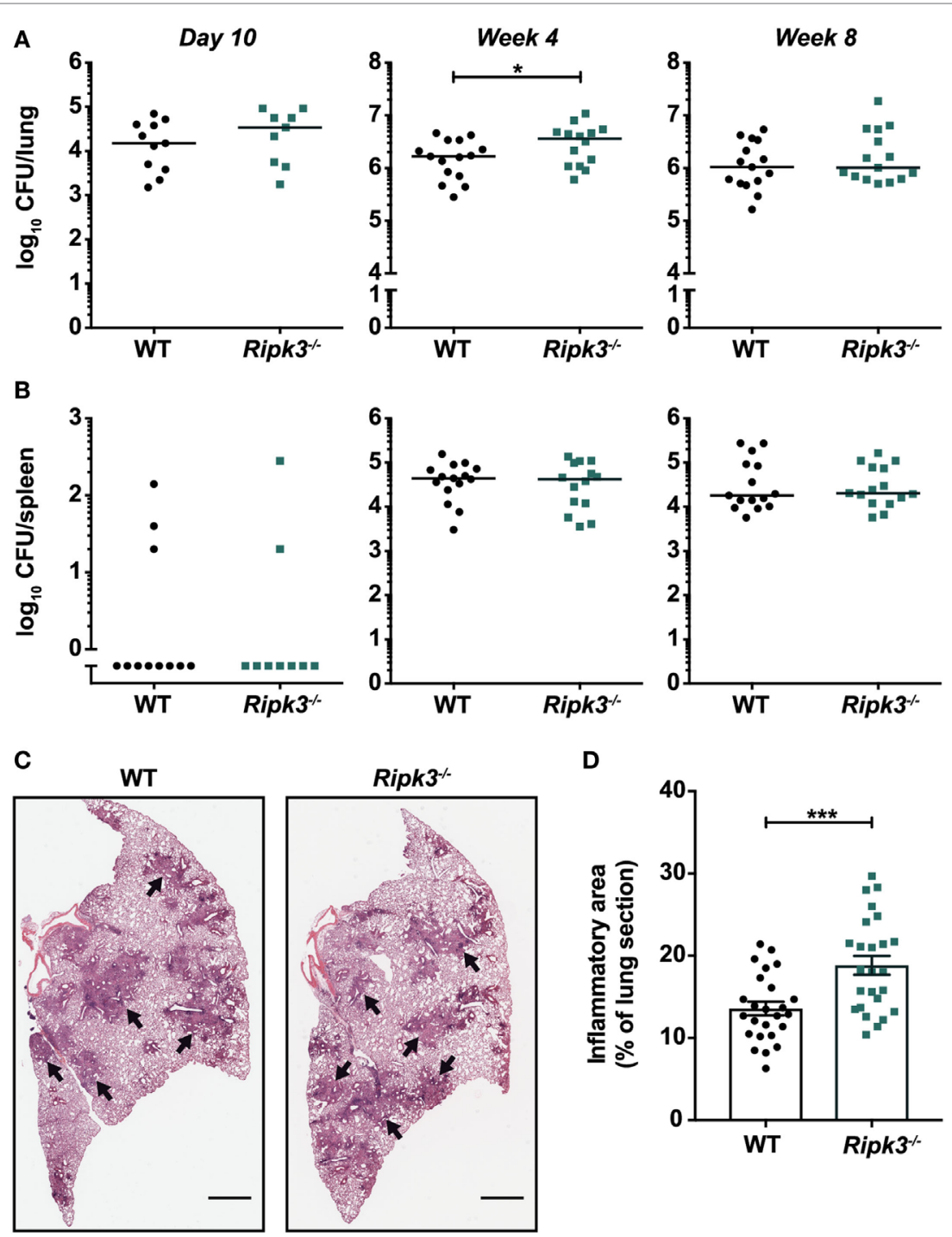

FIGURE 2 | Impact of receptor-interacting protein kinase 3 deficiency on Mycobacterium tuberculosis (Mtb) disease pathogenesis in mice. Bacterial burden in the (A) lungs and (B) spleens of mice at various time points post-infection with aerosolized Mtb. Data were pooled from two (day 10 and week 8) or three (week 4) independent experiments ( $n=9-15$ per time point and genotype). (C) Lung histology of mice 4 weeks post-infection. Black arrows indicate examples of inflammatory areas. Left lobes were stained with hematoxylin and eosin. Representative of 25 mice per genotype. Scale bar represents $1 \mathrm{~mm}$. (D) Quantitation of inflammatory areas in H\&E-stained lung sections of mice 4 weeks post-infection. Data were pooled from five independent experiments $(n=25$ per genotype). Graphs show median (A,B) or mean \pm SEM (D) and each point represents one mouse. There were no statistically significant differences between genotypes $(p>0.05)$ unless indicated. ${ }^{\star} p<0.05 ;{ }^{* \star *} p<0.001$.

points. Interestingly, when a more physiological low-dose aerosol infection model was used, akin to that in the present study, the difference appeared much less marked, more closely reflecting our results. The discrepancy between the intravenous and aerosol models, in terms of the robustness of the difference in bacterial numbers, is intriguing and may reflect the consequences of systemic immune activation arising from intravenous exposure to high doses of $\mathrm{Mtb}$, which may be mitigated to a degree by Ripk3 deletion. Macrophage necrosis induced by RIPK3 was postulated to require the anti-apoptotic protein BCL-XL (25). This could represent an attractive therapeutic target, since several compounds that inhibit this protein are in clinical or pre-clinical trials
$(30,34)$. However, perhaps not surprisingly, given the absence of a phenotype in Ripk $3^{-/-}$mice, we found that neither the conditional deletion of $B c l-x_{L}$ in myeloid cells nor the pharmacologic inhibition of BCL-XL after infection imparted any benefit to mice in terms of reduced bacterial burden. Overall, our data do not support a role for Ripk3 in mediating macrophage death, via necrosis or other forms of cell death. Ripk3 deficiency did not protect mouse macrophages from dying following Mtb infection in vitro, and we observed no difference in macrophage numbers in the lungs or spleens of infected WT and Ripk3 ${ }^{-/-}$mice.

Previous studies addressing RIPK3 function during Mtb infection used either Ripk3 ${ }^{-/-}$BMDMs (as we have done) or RNAi 

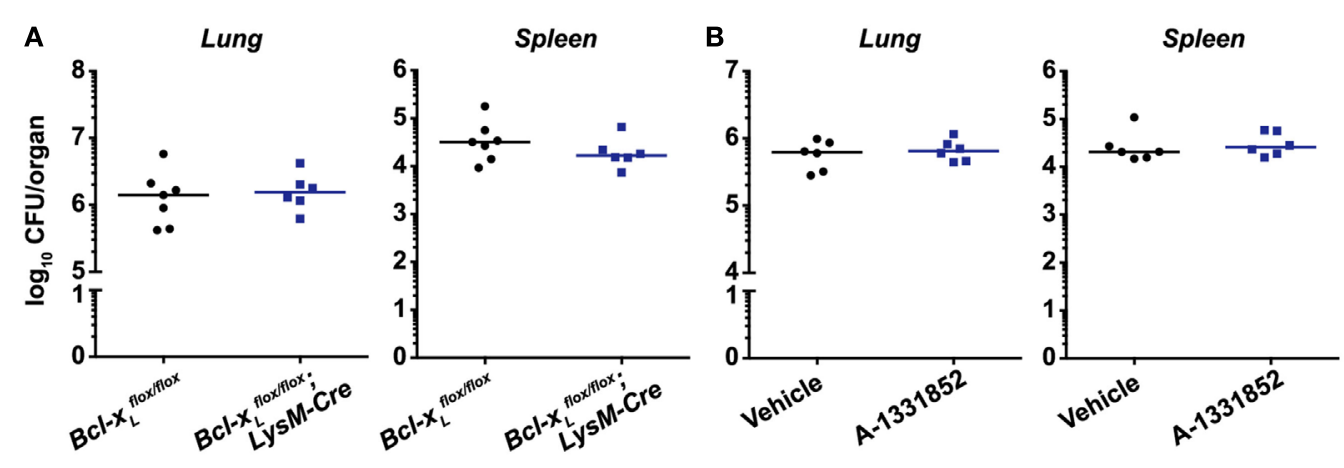

FIGURE 3 | BCL-XL signaling does not contribute to Mycobacterium tuberculosis (Mtb) disease pathogenesis and is not a viable therapeutic target for Mtb infection. (A) Bacterial burden in the lungs and spleens of mice with a conditional deletion of $B c l-x_{L}$ in myeloid cells 4 weeks post-infection with aerosolized Mtb ( $n=6-7$ per genotype). (B) Bacterial burden in the lungs and spleens of mice treated with vehicle or $25 \mathrm{mg} / \mathrm{kg}$ of the BCL-XL-selective inhibitor A-1331852 on 10 days over a 2 -week period, commencing 4 weeks post-infection ( $n=6$ per treatment group). Graphs show median, and each point represents one mouse. There were no statistically significant differences between genotypes $(p>0.05)$.

knockdown of immortalized mouse macrophages (J774A.1) or human monocyte-derived macrophages, all of which were reportedly protected to varying degrees from Mtb-induced death by the loss of RIPK3 $(24,25)$. The source of this discrepancy between our work and these previous studies is unclear. A possible explanation is species differences between human and mouse cells, but this seems unlikely given that one of these studies showed the same phenotype in human macrophages as in mouse BMDMs (25). Although the time point for death analysis was consistent ( $48 \mathrm{~h}$ for mouse macrophages), different MOIs (and potentially means of determining MOI) and methods of quantitating cell death were used, and one study used a different strain of Mtb (24). Nonetheless, we examined death at several MOIs that covered the range used in these previous studies, as well as a validated and sensitive assay for cell death. Zhao et al. (25) correlated the reduced necrotic death of RIPK3-deficient cells with lower intracellular Mtb growth after several days of in vitro culture. Our work showed that RIPK3 did not impact the ability of BMDMs to phagocytose Mtb upon initial exposure. We also assessed intracellular replication of $\mathrm{Mtb}$, but to take into account the totality of factors that impact on bacterial replication in vivo (including other immune cells and cytokines), we performed this analysis on macrophages isolated from the BAL of infected mice. However, we found no difference between genotypes. This is consistent with the absence of any change in the expression of iNOS in Ripk $3^{-/-}$cells, which has been shown to be a major factor restricting Mtb growth in mice (31). This would suggest that any difference in intracellular bacterial numbers in vitro does not translate to infection in vivo. This prior study also reported that RIPK3 promotes ROS production by macrophages, and this was essential to the proposed mechanism for RIPK3-mediated necrosis. We did not examine ROS production as we observed no disparities in cell death or intracellular Mtb burdens between WT and Ripk $3^{-/-}$macrophages, nor any difference in disease outcomes between these genotypes of mice. Our in vivo data, which defines the overall response in the context of all contributors to disease pathogenesis, would suggest that any differences in ROS generation between these two genotypes are mitigated on both a cellular and organism level, such that they do not impact overall disease outcomes.

Although most disease models have identified RIPK3 as a mediator of pathological inflammation, others, including models of influenza A and West Nile virus infection, have highlighted situations in which RIPK3 signaling in fact promotes immunity $(14,35)$. Indeed, we noted a very marginal increase in bacterial numbers and inflammation 4 weeks post-infection with Mtb in mice lacking RIPK3. However, this difference appeared to be transient and was not observed at any other time point, including later stages of infection. Mice begin to control Mtb replication around 3-4 weeks post-infection, coinciding with the development of adaptive immunity $(36,37)$. We speculate that RIPK3 may thus play a small, transient role in regulating protective immunity during the handover between the early innate and the adaptive phases of the immune response. Nevertheless, the increase in bacterial number in $R i p k 3^{-/-}$mice was not borne out of any defect in IL- $1 \beta$ or TNF production in the lungs. The secretion of certain pro-inflammatory cytokines including IL- $1 \beta$ by macrophages and dendritic cells can be induced by RIPK 3 under certain conditions, with Ripk3 deletion attenuating inflammasome activation and IL- $1 \beta$ processing following LPS stimulation or infection with certain RNA viruses (9-11). However, we did not observe any defect in IL- $1 \beta$ production by Ripk $3^{-/-}$macrophages infected with Mtb, consistent with our in vivo data. This may be due to the fact that the cellular inhibitor of apoptosis and X-linked inhibitor of apoptosis proteins restrict inflammasome activation by RIPK3 and must themselves be inhibited/deleted to liberate this aspect of RIPK3 signaling $(8,9,11,12)$. It is thus unclear why Ripk3 $3^{-/-}$ mice had a mild and transient impairment in Mtb control at 4 weeks post-infection. However, RIPK3 interacts with other signaling molecules including RIPK1, and this interaction has been shown to regulate multiple signaling pathways such as ERK, cFos, and NF- $\kappa$ B (13), and potentially JNK (38), leading to changes in inflammatory responses. In fact, RIPK3 has been implicated in the production of several cytokines in addition to IL-1 $\beta$ during host responses to pathogens, including type I IFN 

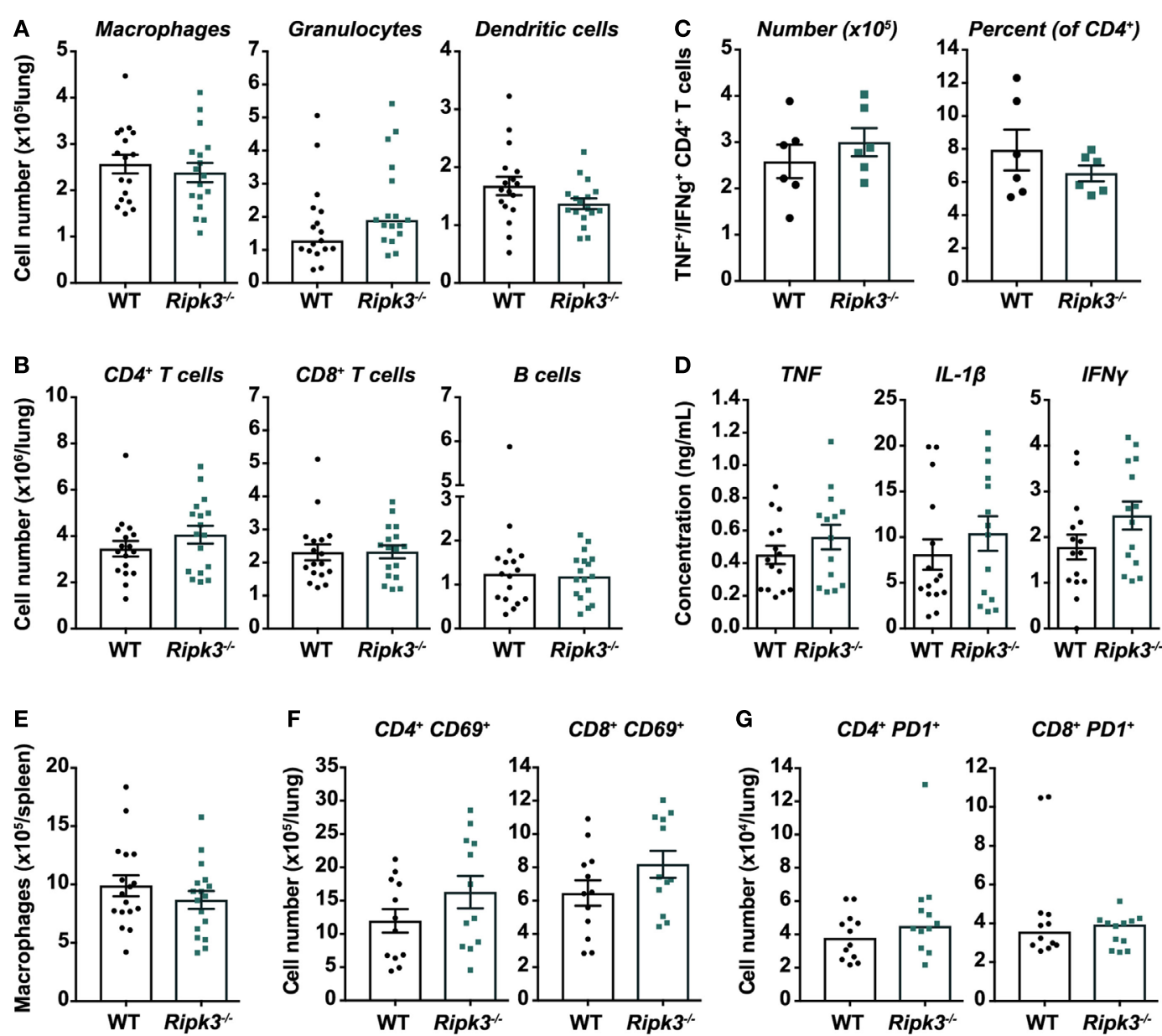

FIGURE 4 | Immune responses in Mycobacterium tuberculosis (Mtb)-infected mice are unaltered by receptor-interacting protein kinase 3 deficiency. Total number of (A) myeloid and (B) lymphoid immune cells in the lungs of mice infected with aerosolized Mtb. Flow cytometry was used to identify macrophages/ monocytes (CD11 b+ Gr1 $\left.{ }^{\text {low }}\right)$, granulocytes (CD11 b+ Gr1 $\left.{ }^{\text {high }}\right)$, dendritic cells (CD11 $\left.\mathrm{c}^{+} \mathrm{MHCll}^{+}\right), \mathrm{CD}^{+}$and $\mathrm{CD} 8^{+} \mathrm{T}$ cells, and B cells $\left(\mathrm{CD} 19^{+} \mathrm{MHCl}+\mathrm{I}^{+}\right)$. Data were pooled from three independent experiments ( $n=17$ per genotype). (C) Number of Mtb early secreted antigenic target 6 (ESAT-6)-specific CD4+ ${ }^{+}$cells in the lungs of Mtb-infected mice. Cells were isolated from the lungs and stimulated ex vivo with Mtb ESAT- 6 peptide, and polyfunctional (TNF ${ }^{+}$IFN $\gamma^{+}$) CD4 ${ }^{+} \mathrm{T}$ cells identified by intracellular cytokine staining and flow cytometry. Representative of two independent experiments ( $n=6$ per genotype and experiment). (D) Concentrations of TNF, IL-1 $\beta$, and IFN $\gamma$ in lung homogenates from infected mice, quantitated by ELISA. Data were pooled from three independent experiments ( $n=14-15$ per genotype). (E) Number of macrophages in the spleens of wild-type and Ripk3 ${ }^{-/-}$mice 4 weeks post-infection with aerosolized Mtb. Data were pooled from three independent experiments $\left(n=17\right.$ per genotype). Number of (F) acutely $\left(\right.$ CD69+ ${ }^{+}$and $(\mathbf{G})$ chronically $\left(\right.$ PD $\left.1^{+}\right)$activated CD4 $^{+}$ and $\mathrm{CD}^{+} \mathrm{T}$ cells in the lungs of infected mice. Data were pooled from two independent experiments $(n=12$ per genotype). All analyses (A-G) were performed at 4 weeks post-infection with aerosolized Mtb. Graphs show median or mean \pm SEM, and each point represents one mouse. There were no statistically significant differences between genotypes $(p>0.05)$

$(14,15)$ and chemokines (35), from immune and non-immune cell types, respectively. We hypothesize that perhaps deficiencies in such cytokines in Ripk $3^{-/-}$mice at the critical interphase between innate and adaptive immunity may have conspired to produce the observed phenotype.

Collectively, our findings indicate that in the context of Mtb infection, RIPK3 does not play a fundamental role in regulating inflammatory responses or necrotic macrophage death in vivo, at least not in a non-redundant capacity. We would, therefore, argue against the pursuit of RIPK3 inhibition as a tractable therapeutic option for TB.

\section{ETHICS STATEMENT}

The Walter and Eliza Hall Institute of Medical Research Animal Ethics Committee reviewed and approved all animal experiments.

\section{AUTHOR CONTRIBUTIONS}

MS, SO, and MP conceived and designed the research. MS, SO, and GE performed the experiments and analyzed the data. MS and MP wrote the manuscript. All authors contributed to manuscript revision, read, and approved the submitted version. 


\section{ACKNOWLEDGMENTS}

We thank Vishva Dixit (Genentech) for Ripk $3^{-/-}$mice, James Vince (WEHI) for $\mathrm{Bcl}-x_{L}^{f l o x} / f l o x ; L y s M-C r e$ mice, and Lachlan Whitehead and the Centre for Dynamic Imaging (WEHI) for assistance with image analysis.

\section{FUNDING}

This work was supported by National Health and Medical Research Council Australia (Grants 1006592, 1045549, and 1065626 to MP

\section{REFERENCES}

1. Zhang D, Lin J, Han J. Receptor-interacting protein (RIP) kinase family. Cell Mol Immunol (2010) 7:243-9. doi:10.1038/cmi.2010.10

2. Silke J, Rickard JA, Gerlic M. The diverse role of RIP kinases in necroptosis and inflammation. Nat Immunol (2015) 16:689-97. doi:10.1038/ni.3206

3. Humphries F, Yang S, Wang B, Moynagh PN. RIP kinases: key decision makers in cell death and innate immunity. Cell Death Differ (2015) 22: 225-36. doi:10.1038/cdd.2014.126

4. Zhao J, Jitkaew S, Cai Z, Choksi S, Li Q, Luo J, et al. Mixed lineage kinase domain-like is a key receptor interacting protein 3 downstream component of TNF-induced necrosis. Proc Natl Acad Sci U S A (2012) 109:5322-7. doi:10.1073/pnas.1200012109

5. Sun L, Wang H, Wang Z, He S, Chen S, Liao D, et al. Mixed lineage kinase domain-like protein mediates necrosis signaling downstream of RIP3 kinase. Cell (2012) 148:213-27. doi:10.1016/j.cell.2011.11.031

6. Stutz MD, Ojaimi S, Allison C, Preston S, Arandjelovic P, Hildebrand JM, et al. Necroptotic signaling is primed in Mycobacterium tuberculosis-infected macrophages, but its pathophysiological consequence in disease is restricted. Cell Death Differ (2018) 25:951-65. doi:10.1038/s41418-017-0031-1

7. Newton K, Dugger DL, Maltzman A, Greve JM, Hedehus M, MartinMcNulty B, et al. RIPK3 deficiency or catalytically inactive RIPK1 provides greater benefit than MLKL deficiency in mouse models of inflammation and tissue injury. Cell Death Differ (2016) 23:1565-76. doi:10.1038/cdd. 2016.46

8. Lawlor KE, Feltham R, Yabal M, Conos SA, Chen KW, Ziehe S, et al. XIAP loss triggers RIPK3- and caspase-8-driven IL-1 $\beta$ activation and cell death as a consequence of TLR-MyD88-induced cIAP1-TRAF2 degradation. Cell Rep (2017) 20:668-82. doi:10.1016/j.celrep.2017.06.073

9. Vince JE, Wong WW-L, Gentle I, Lawlor KE, Allam R, O’Reilly L, et al. Inhibitor of apoptosis proteins limit RIP3 kinase-dependent interleukin-1 activation. Immunity (2012) 36:215-27. doi:10.1016/j.immuni.2012.01.012

10. Wang X, Jiang W, Yan Y, Gong T, Han J, Tian Z, et al. RNA viruses promote activation of the NLRP3 inflammasome through a RIP1-RIP3-DRP1 signaling pathway. Nat Immunol (2014) 15:1126-33. doi:10.1038/ni.3015

11. Lawlor KE, Khan N, Mildenhall A, Gerlic M, Croker BA, D'Cruz AA, et al. RIPK3 promotes cell death and NLRP3 inflammasome activation in the absence of MLKL. Nat Commun (2015) 6:6282. doi:10.1038/ncomms7282

12. Wong WW-L, Vince JE, Lalaoui N, Lawlor KE, Chau D, Bankovacki A, et al. cIAPs and XIAP regulate myelopoiesis through cytokine production in an RIPK1- and RIPK3-dependent manner. Blood (2014) 123:2562-72. doi:10.1182/blood-2013-06-510743

13. Najjar M, Saleh D, Zelic M, Nogusa S, Shah S, Tai A, et al. RIPK1 and RIPK3 kinases promote cell-death-independent inflammation by toll-like receptor 4. Immunity (2016) 45:46-59. doi:10.1016/j.immuni.2016.06.007

14. Downey J, Pernet E, Coulombe F, Allard B, Meunier I, Jaworska J, et al. RIPK3 interacts with MAVS to regulate type I IFN-mediated immunity to influenza A virus infection. PLoS Pathog (2017) 13:e1006326. doi:10.1371/ journal.ppat.1006326

15. Saleh D, Najjar M, Zelic M, Shah S, Nogusa S, Polykratis A, et al. Kinase activities of RIPK1 and RIPK3 can direct IFN- $\beta$ synthesis induced by lipopolysaccharide. J Immunol (2017) 198:4435-47. doi:10.4049/jimmunol.1601717 and 1056282 to SO). The Sylvia \& Charles Viertel Senior Medical Research Fellowship (MP), the Victorian State Government Operational Infrastructure Support, and the Independent Research Institutes Infrastructure Support Scheme of the Australian Government National Health and Medical Research Council.

\section{SUPPLEMENTARY MATERIAL}

The Supplementary Material for this article can be found online at https://www.frontiersin.org/articles/10.3389/fimmu.2018.01178/ full\#supplementary-material.

16. Kataoka K, Matsumoto H, Kaneko H, Notomi S, Takeuchi K, Sweigard JH, et al. Macrophage- and RIP3-dependent inflammasome activation exacerbates retinal detachment-induced photoreceptor cell death. Cell Death Dis (2015) 6:e1731-1731. doi:10.1038/cddis.2015.73

17. Dorhoi A, Yeremeev V, Nouailles G, Weiner J, Jörg S, Heinemann E, et al. Type I IFN signaling triggers immunopathology in tuberculosis-susceptible mice by modulating lung phagocyte dynamics. Eur J Immunol (2014) 44:2380-93. doi:10.1002/eji.201344219

18. Manca C, Tsenova L, Freeman S, Barczak AK, Tovey M, Murray PJ, et al. Hypervirulent $M$. tuberculosis W/Beijing strains upregulate type I IFNs and increase expression of negative regulators of the Jak-Stat pathway. J Interferon Cytokine Res (2005) 25:694-701. doi:10.1089/jir.2005.25.694

19. Berry MPR, Graham CM, McNab FW, Xu Z, Bloch SAA, Oni T, et al. An interferon-inducible neutrophil-driven blood transcriptional signature in human tuberculosis. Nature (2010) 466:973-7. doi:10.1038/nature09247

20. Bourigault M-L, Segueni N, Rose S, Court N, Vacher R, Vasseur V, et al. Relative contribution of IL- $1 \alpha$, IL- $1 \beta$ and TNF to the host response to Mycobacterium tuberculosis and attenuated M. bovis BCG. Immun Inflamm Dis (2013) 1:47-62. doi:10.1002/iid3.9

21. Mayer-Barber KD, Andrade BB, Barber DL, Hieny S, Feng CG, Caspar P, et al. Innate and adaptive interferons suppress IL- $1 \alpha$ and IL- $1 \beta$ production by distinct pulmonary myeloid subsets during Mycobacterium tuberculosis infection. Immunity (2011) 35:1023-34. doi:10.1016/j.immuni.2011.12.002

22. Dorhoi A, Nouailles G, Jörg S, Hagens K, Heinemann E, Pradl L, et al. Activation of the NLRP3 inflammasome by Mycobacterium tuberculosis is uncoupled from susceptibility to active tuberculosis. Eur J Immunol (2012) 42:374-84. doi:10.1002/eji.201141548

23. Zhang G, Zhou B, Li S, Yue J, Yang H, Wen Y, et al. Allele-specific induction of IL-1 $\beta$ expression by C/EBP $\beta$ and PU.1 contributes to increased tuberculosis susceptibility. PLoS Pathog (2014) 10:e1004426. doi:10.1371/journal. ppat.1004426

24. Butler RE, Krishnan N, Garcia-Jimenez W, Francis R, Martyn A, Mendum T, et al. Susceptibility of Mycobacterium tuberculosis-infected host cells to phospho-MLKL driven necroptosis is dependent on cell type and presence of TNF $\alpha$. Virulence (2017) 64:1-13. doi:10.1080/21505594.2017.1377881

25. Zhao X, Khan N, Gan H, Tzelepis F, Nishimura T, Park S-Y, et al. Bcl-xL mediates RIPK3-dependent necrosis in M. tuberculosis-infected macrophages. Mucosal Immunol (2017) 8:668. doi:10.1038/mi.2017.12

26. Fayaz SM, Suvanish Kumar VS, Davis CK, Rajanikant GK. Novel RIPK3 inhibitors discovered through a structure-based approach exert post-ischemic neuroprotection. Mol Divers (2016) 20:719-28. doi:10.1007/s11030-0169663-1

27. Degterev A, Linkermann A. Generation of small molecules to interfere with regulated necrosis. Cell Mol Life Sci (2016) 73:2251-67. doi:10.1007/ s00018-016-2198-x

28. Newton K, Sun X, Dixit VM. Kinase RIP3 is dispensable for normal NFkappa Bs, signaling by the B-cell and T-cell receptors, tumor necrosis factor receptor 1, and toll-like receptors 2 and 4. Mol Cell Biol (2004) 24:1464-9. doi:10.1128/MCB.24.4.1464-1469.2004

29. Speir M, Lawlor KE, Glaser SP, Abraham G, Chow S, Vogrin A, et al. Eliminating Legionella by inhibiting BCL-XL to induce macrophage apoptosis. Nat Microbiol (2016) 1:15034. doi:10.1038/nmicrobiol.2015.34 
30. Leverson JD, Phillips DC, Mitten MJ, Boghaert ER, Diaz D, Tahir SK, et al. Exploiting selective BCL-2 family inhibitors to dissect cell survival dependencies and define improved strategies for cancer therapy. Sci Transl Med (2015) 7:ra40-279. doi:10.1126/scitranslmed.aaa4642

31. MacMicking JD, North RJ, LaCourse R, Mudgett JS, Shah SK, Nathan CF. Identification of nitric oxide synthase as a protective locus against tuberculosis. Proc Natl Acad Sci U S A (1997) 94:5243-8. doi:10.1073/pnas. 94.10.5243

32. Bean AG, Roach DR, Briscoe H, France MP, Korner H, Sedgwick JD, et al. Structural deficiencies in granuloma formation in TNF gene-targeted mice underlie the heightened susceptibility to aerosol Mycobacterium tuberculosis infection, which is not compensated for by lymphotoxin. J Immunol (1999) 162:3504-11. doi:10.1128/mBio.01686-16

33. Zhang H, Nimmer PM, Tahir SK, Chen J, Fryer RM, Hahn KR, et al. Bcl-2 family proteins are essential for platelet survival. Cell Death Differ (2007) 14: 943-51. doi:10.1038/sj.cdd.4402081

34. Opferman JT. Attacking cancer's Achilles heel: antagonism of anti-apoptotic BCL-2 family members. FEBS J (2016) 283:2661-75. doi:10.1111/febs.13472

35. Daniels BP, Snyder AG, Olsen TM, Orozco S, Oguin TH, Tait SWG, et al. RIPK3 restricts viral pathogenesis via cell death-independent neuroinflammation. Cell (2017) 169:301-13.e11. doi:10.1016/j.cell.2017.03.011
36. Mogues T, Goodrich ME, Ryan L, LaCourse R, North RJ. The relative importance of $\mathrm{T}$ cell subsets in immunity and immunopathology of airborne Mycobacterium tuberculosis infection in mice. J Exp Med (2001) 193:271-80. doi:10.1084/jem.193.3.271

37. Rhoades ER, Frank AA, Orme IM. Progression of chronic pulmonary tuberculosis in mice aerogenically infected with virulent Mycobacterium tuberculosis. Tuber Lung Dis (1997) 78:57-66. doi:10.1016/S0962-8479(97)90016-2

38. Christofferson DE, Li Y, Hitomi J, Zhou W, Upperman C, Zhu H, et al. A novel role for RIP1 kinase in mediating TNF $\alpha$ production. Cell Death Dis (2012) 3:e320. doi:10.1038/cddis.2012.64

Conflict of Interest Statement: The authors declare that the research was conducted in the absence of any commercial or financial relationships that could be construed as a potential conflict of interest.

Copyright (C) 2018 Stutz, Ojaimi, Ebert and Pellegrini. This is an open-access article distributed under the terms of the Creative Commons Attribution License (CC BY). The use, distribution or reproduction in other forums is permitted, provided the original author(s) and the copyright owner are credited and that the original publication in this journal is cited, in accordance with accepted academic practice. No use, distribution or reproduction is permitted which does not comply with these terms. 